\title{
Curvature and Non-Resonance Compton Gamma-Ray Emission of a Radio Pulsar with a Non-Dipolar Magnetic Field
}

\author{
D. P. Barsukov, E. M. Kantor, and A. I. Tsygan \\ Ioffe Physical Technical Institute, St. Petersburg, Russia \\ Received March 15, 2006; in final form, December 27, 2006
}

\begin{abstract}
We consider the influence of a non-dipolar magnetic field on the gamma-ray emission from the polar regions of a radio pulsar. The pulsar is treated in a Goldreich-Julian model with a free flow of charge from the surface of the neutron star. When finding the intensity of the gamma-ray radiation of the pulsar tube, both curvature gamma-ray radiation from the primary electrons and non-resonance inverse Compton scattering of thermal photons from the polar cap on primary electrons are taken into account. When finding the height of the upper plate of the pulsar diode, we included only positrons created by the curvature radiation of primary electrons. We assumed that the polar cap is heated by the return positron current. The influence on the gamma-ray emission of variations in both the radius of curvature of the magnetic force lines and in the electric field due to the non-dipolarity of the magnetic field were taken into account. The presence of even weak non-dipolarity of the magnetic field leads to a sharp decrease in the intensity of the gamma-ray emission from the pulsar tube at energies $1-100 \mathrm{MeV}$, while the intensity of the inverse Compton radiation (at energies $1-100 \mathrm{GeV}$ ) varies only relatively weakly.
\end{abstract}

PACS numbers : 97.60.Gb, 95.30.Gv, 95.30.Qd

DOI: $10.1134 / \mathrm{S} 1063772907060054$

\section{INTRODUCTION}

Possibilities for studying and observing the formation of small-scale magnetic fields on neutron stars are being actively discussed in the literature [1-5]. The presence of a small-scale magnetic field could affect the operation of radio pulsars, including the intensity of gamma-ray emission from the pulsar tube. The gamma-ray emission of pulsars in the absence of small-scale magnetic fields, with a purely dipolar magnetic field, has been considered in numerous studies (see, for example, [6-10]). The influence of a no-dipolar magnetic field has been considered in $[6$, $11,12]$

The current work is a continuation of the studies [13-15]. When considering the influence of a nondipolar magnetic field on the operation of a radio pulsar, we use the model developed in [15-17]. This model takes into account both the increase in curvature of the force lines and the effect of the nondipolar magnetic field on the electrical potential when determining the characteristics of the pulsar gammaray emission.

The radio pulsar is treated in a Golreich-Julian model with a free outflow of electrons from the neutron-star surface. The pulsar diode is located at the surface of the neutron star (a "polar cap" model).
Only curvature radiation by ultra-relativistic primary electrons and radiation associated with nonresonance Compton scattering of thermal photons from the polar cap on primary electrons are taken into account when calculating the gamma-ray emission of a radio pulsar. We neglect non-resonance Compton radiation of photons from the remainder of the neutron-star surface, which is fully justified for neutron stars that have already had time to cool to fairly low temperatures. We likewise do not include processes associated with resonance inverse Compton scattering, and we neglect radiation by secondary electrons and positrons.

Only the absorption of photons in the magnetic field associated with the creation of electron-positron pairs in an unbound state are taken into account; we neglect the contribution to the cross section of corrections associated with the polarization of the vacuum in the magnetic field, as well as radiative corrections. The calculations of the creation of electronpositron pairs take into account only their creation in the magnetic field from photons generated via curvature radiation by the primary electrons. We neglect all general-relativistic effects except the inertial framedragging effect, which makes a substantial contribution to the electrostatic potential. 


\section{ELECTRIC FIELD}

We will describe the non-dipolarity of the magnetic field and calculate the accelerating electrical potential using the same model that was used in [13] (see also $[15,17])$.

Let a neutron star with radius $a$ have a magnetic dipole moment $\mathbf{m}$, with the angle between $\mathbf{m}$ and the axis of rotation of the pulsar $\boldsymbol{\Omega}$ being $\chi$. Another magnetic dipole with moment $\mathbf{m}_{1}$ is located at a depth $a \Delta$ from the surface in the region of the magnetic pole of the neutron star. The dipole moment $\mathbf{m}_{1}$ is perpendicular to $\mathbf{m}$; we denote the angle between $\mathbf{m}_{1}$ and the $(\boldsymbol{\Omega}, \mathbf{m})$ plane $\alpha(\alpha=\pi$ corresponds to the case when $\mathbf{m}_{1}$ lies in the $(\boldsymbol{\Omega}, \mathbf{m})$ plane and is directed toward the rotational axis of the pulsar). We will henceforth take the parameter $\Delta$ to be equal to 0.1 .

We introduce a spherical coordinate system $(\eta=$ $r / a, \theta, \phi)$ with its origin $\eta=0$ at the center of the neutron star, the $O z$ axis directed along $\mathbf{m}$, and the $O x$ axis directed along $\mathbf{m}_{1}$, so that $\mathbf{m}=m \mathbf{e}_{z}$ and $\mathbf{m}_{1}=m_{1} \mathbf{e}_{x}$. The magnetic-field strength can be found using the expression [13]

$$
\begin{gathered}
B_{\eta}=\frac{B_{0}}{\eta^{3}}, \quad B_{\theta}=\frac{B_{0}}{\eta^{3}}\left(\frac{\theta}{2}+\mu \cos \phi\right), \\
B_{\phi}=-\frac{B_{0}}{\eta^{3}} \mu \sin \phi
\end{gathered}
$$

where $\mu=\nu\left(\frac{\Delta \eta}{\eta-1+\Delta}\right)^{3}, \nu=B_{1} / B_{0}$, and $B_{0}$ and $B_{1}$ are the strengths of the magnetic fields of the dipoles $\mathbf{m}$ and $\mathbf{m}_{1}$ at the magnetic pole of the neutron $\operatorname{star}(\eta=1, \theta=0, \phi=0)$. We use formula (2) of [13] to find the radius of curvature of the magnetic-field lines.

When obtaining the electric field, we take the Goldreich-Julian density to be

$$
\begin{gathered}
\rho_{\text {eff }}=\frac{\Omega B_{0}}{2 \pi c} \frac{1}{\eta^{3}} \\
\times\left[\left(1-\frac{k}{\eta^{3}}\right) \cos \chi+\mu\left(1+\frac{1}{2} \frac{k}{\eta^{3}}\right) \sin \chi \cos \alpha\right],
\end{gathered}
$$

where $\Omega=\frac{2 \pi}{P}, P$ is the rotational period of the pulsar, and $k$ is the coefficient associated with the general relativistic inertial frame-dragging effect [17, 18], which we will take everywhere to be $k=0.15$.

As in [13], we assume $\left.E_{\|}\right|_{\eta=1}=0$ and $\left.\Phi\right|_{\eta=1}=$ 0 at the surface of the neutron star, with $\Phi=0$ at the walls of the pulsar tube. We assume the upper plate of the diode to be at a height $z_{c} \equiv \eta_{c}-1$ (where the amount of created electron-positron plasma is sufficient to screen the electric field); i.e., $\left.E_{\|}\right|_{\eta=\eta_{c}}=0$.
The electric potential is treated using formula (6) of [13], which in our case has the form

$$
\begin{gathered}
\Phi=2 \Phi_{0} K_{1} \sum_{i=0}^{\infty} \frac{1}{\gamma_{i}^{2}}\left(z+\frac{F\left(z, \gamma_{i}\right)}{\gamma_{i}}\right) \\
\times \frac{2}{k_{i} J_{1}\left(k_{i}\right)} J_{0}\left(k_{i} \xi\right), \\
\rho=\frac{\Omega B}{2 \pi c} A(\xi),
\end{gathered}
$$

where

$$
\begin{gathered}
F(z, \gamma)=-\frac{\left(1-e^{-\gamma z}\right)\left(1+e^{-\gamma\left(z_{c}-z\right)}\right)}{1+e^{-\gamma z_{c}}}, \\
A(\xi)=-K_{0}-K_{1} \\
\times \sum_{i=0}^{\infty} \frac{1}{\gamma_{i}} \frac{\left(1-e^{-\gamma_{i} z_{c}}\right)}{\left(1+e^{-\gamma_{i} z_{c}}\right)} \frac{2}{k_{i} J_{1}\left(k_{i}\right)} J_{0}\left(k_{i} \xi\right),
\end{gathered}
$$

$\Phi_{0}=\frac{\Omega a}{c} B_{\text {sur }} a, \gamma_{i}=k_{i} / \theta_{\text {sur }}, k_{i}$ is the root of the equation $J_{0}(k)=0, \quad B_{\text {sur }}$ is the magnetic-field strength at the surface of the neutron star at the center of the tube $(\eta=1, \xi=0, \phi=0)$, and $\theta_{\text {sur }} a$ is the radius of the pulsar tube at the neutronstar surface, which is found from the conservation of magnetic flux in the tube, $\theta_{\text {sur }}^{2} a^{2} B_{\text {sur }}=\theta_{0}^{2} a^{2} B_{0}$, where $\theta_{0}=\sqrt{\frac{\Omega a}{c}}$ (see formula (5) in [16]).

The expressions for the coefficients $K_{0}$ and $K_{1}$ have the form

$$
\begin{gathered}
K_{0}=\frac{1}{\sqrt{1+\nu^{2}}}\left[(1-k) \cos \chi+\left(1+\frac{k}{2}\right)\right. \\
\times \nu \sin \chi \cos \alpha], \\
K_{1}=\frac{1}{\sqrt{1+\nu^{2}}}\left[3 k+\frac{3 \nu}{\Delta} \frac{\nu}{1+\nu^{2}}(1-\Delta)\right. \\
\times(1-k)] \cos \chi \\
-\frac{3 \nu}{\Delta} \frac{1}{\left(1+\nu^{2}\right)^{3 / 2}}\left[1-\Delta+\frac{k}{2}+\frac{k}{2} \nu^{2} \Delta\right] \sin \chi \cos \alpha
\end{gathered}
$$

\section{GENERATION OF GAMMA-RAY EMISSION}

We treat the generation of gamma-ray emission in the same way as is done in [13]. We take the energy of the primary electrons at the point $x=(\eta, \xi, \phi)$ to be $E=m c^{2} \Gamma=e \Phi+m c^{2}$, where $\Phi=\Phi(\eta, \xi, \phi)$ is the potential (3) at $x, m$ is the electron mass, $c$ is the speed of light, and $e$ is the absolute value of the electron charge. In the case of a purely dipolar field

ASTRONOMY REPORTS Vol.51 No.6 2007 
( $\nu=0)$ with $z \geq z_{c}$, the energy of the electrons is calculated as follows [14]:

$$
\Gamma(z, \xi, \phi)=\frac{\Gamma_{c}}{\left[1+\frac{9}{8} \frac{r_{e}}{a} \theta_{0}^{2} \xi^{2} \Gamma_{c}^{3} \log \left(\frac{\eta}{\eta_{c}}\right)\right]^{1 / 3}}, .
$$

where $\Gamma_{c}=\frac{e \Phi}{m c^{2}}\left(z_{c}, \xi, \phi\right)+1$ and we have introduced the notation $r_{e}=\frac{e^{2}}{m c^{2}}$ for the classical radius of the electron. Radiative damping is taken into account only for a purely dipolar magnetic field; in other cases, there is only a small amount of radiative cooling due to curvature radiation.

The height of the gap $z_{c}$ is determined in the same way as in [13]; i.e., it is chosen so that the multiplicative coefficient $Q$ is equal to 0.1 at the point $(z=$ $\left.z_{c}, \xi=0.5, \phi=0\right)$. When finding $Q$, we include the creation of electron-positron pairs in the magnetic field only by curvature-radiation photons generated by primary electrons.

The return positron current is calculated using Eq. (3) of [15], which, in the case of a purely dipolar field $(\nu=0)$, takes the form

$$
n_{+}=\frac{k}{2}\left(1-\frac{k}{\eta_{c}^{3}}\right) n_{e}
$$

in the case of a non-dipolar field, we use the expression

$$
n_{+}=\frac{K_{1}}{2} z_{c} n_{e}
$$

where $n_{+}$is the number density of positrons in the return current and $n_{e}$ is the number density of primary electrons.

We estimate the temperature of the polar hot spot using the formula

$$
\sigma T^{4}=n_{+} c m c^{2} \Gamma,
$$

where $\Gamma$ is the energy of the primary electrons at the point ( $z=z_{c}, \xi=0, \phi=0$ ), and $n_{+}$is the number density of positrons in the return current at the point $(z=0, \xi=0, \phi=0)$.

The absorption of photons in the magnetic field with the creation of electron-positron pairs is taken into account in the same way as in [13].

The intensity of the curvature radiation is likewise calculated as in [13]. We neglect the contribution of processes associated with resonance inverse Compton scattering to the gamma-ray emission. The intensity of gamma-rays associated with non-resonance inverse Compton scattering of thermal photons from the hot spot at the polar cap is calculated as follows.

At each point $x$, we introduce a coordinate system $K$ that is fixed to the neutron star (pulsar tube), with the $O z$ axis directed along the magnetic field $\mathbf{B}(x)$, i.e., $\mathbf{B}(x)=B(x) \mathbf{e}_{z}$, and a coordinate system $K^{\prime}$ in which the electrons are at rest (at $x$ ), with the coordinate axes coincident with those for $K$. We will denote quantities measured in $K^{\prime}$ with a prime and those associated with scattered photons with the subscript $s$.

We take the distribution function for the primary electrons in the coordinate system $K$ to have the form

$$
f_{e}(\mathbf{x}, \mathbf{p})=n_{e}(x) \delta^{3}\left(\mathbf{p}-m c \Gamma \beta \mathbf{e}_{z}\right),
$$

where $n_{e}(x)$ is the number density of primary electrons at $x$ and $\beta=\frac{\sqrt{\Gamma^{2}-1}}{\Gamma}$ is their velocity at this point.

The distribution function for the thermal photons from the hot spot $f_{\gamma}$ at $x$ is specified as follows. Let $x_{c}$ be the center of the polar hot spot (the point where the central magnetic-field line $\xi=0$ intersects the surface of the neutron star) and $d_{c}=\theta_{\text {sur }} a$ be its radius. We also introduce the vector $\mathbf{d}=\mathbf{x}-\mathbf{x}_{c}$ and the angle $\theta_{c}$ such that $\tan \theta_{c}=d_{c} / d$.

We consider at $x$ a coordinate system $K_{d}$ that is at rest in $K$, with the $O z_{d}$ axis directed along d; i.e., $\mathbf{d}=d \mathbf{e}_{z_{d}}$. We will take the distribution function for photons from the hot spot $f_{\gamma}$ at $x$ in the coordinate system $K_{d}$ to have the form [8]

$$
f_{\gamma}=n_{\gamma} \frac{\delta\left(\gamma-\gamma_{c}\right)}{\gamma_{c}^{2}} \theta\left(\mu_{d}-\mu_{c}\right) \frac{1}{2 \pi}
$$

i.e., we adopt $\delta\left(\gamma-\gamma_{c}\right)$ in place of a Planck spectrum. Here, $d N=f_{\gamma} \gamma^{2} d \gamma d \Omega_{\gamma} d V$ is the number of photons in volume $d V$ with energy $\gamma$ (in units of $m c^{2}$ ) in the interval $d \gamma$ with momenta $\mathbf{p}$ lying within the solid angle $d \Omega_{\gamma}=d \mu_{d} d \phi_{d} ; \mu_{d}=\cos \theta_{d}$, where $\theta_{d}$ is the angle between $\mathbf{p}$ and the $O z_{d}$ axis; $\theta(x)$ is the Heavyside function; $n_{\gamma}=20 \mathrm{~cm}^{-3}\left(\frac{T}{1 \mathrm{~K}}\right)^{3}$; and the quantity $\gamma_{c}$ is chosen as in [8], $\gamma_{c}=2.7 \frac{k_{B} T}{m c^{2}}$, where $T$ is the temperature of the hot spot.

We calculate the intensity of the gamma-ray emission using the approximation developed in $[8,19]$. In particular, we make the following assumptions:

(a) $\Gamma \gg 1$, and we can neglect all terms of order $\frac{1}{\Gamma} \ll 1$

(b) in $K^{\prime}$, the distribution of photons incident on the electrons can be taken to have the form $f_{\gamma}^{\prime} \sim$ $\delta\left(\mu^{\prime}+1\right)$; in particular, we assume $\Psi^{\prime}=\pi-\theta_{s}^{\prime}$;

(c) $\gamma \ll \gamma_{s}<\Gamma \beta$ and $\gamma \ll 1$.

Here, $\gamma$ is the energy of a photon colliding with an electron and $\gamma_{s}$ is the energy of the scattered photon 
Height of the upper diode plate $z_{c}$, energy of primary electrons $\Gamma$ at $\left(z=z_{c}, \xi=0.5, \phi=0\right)$ in units of $m c^{2}$, and the temperature $T$ of the hot spot used to calculate the gamma-ray intensity for pulsars with $B=0.02 B_{c r}$, $P=0.2 \mathrm{~s}, \chi=10^{\circ}, \Delta=0.1, k=0.15$

\begin{tabular}{c|c|c|c}
\hline$\nu$ & $z_{c}, 10^{-2}$ & $\Gamma, 10^{6}$ & $T, 10^{6} \mathrm{~K}$ \\
\hline 0 & 29.6 & 33.9 & 3.67 \\
0.1 & 3.2 & 2.95 & 1.64 \\
0.2 & 2.05 & 1.81 & 1.53 \\
0.3 & 1.61 & 1.53 & 1.55 \\
\multicolumn{3}{|c|}{$\alpha=\frac{\pi}{2}$} \\
0.1 & 4.28 & 3.22 & 1.80 \\
0.2 & 2.53 & 1.86 & 1.63 \\
0.3 & 1.89 & 1.52 & 1.61 \\
\hline
\end{tabular}

in the $K$ system (measured in units of $m c^{2}$ ). We also denote $\mathbf{p}$ and $\mathbf{p}_{s}$ to be the momenta of the incident and scattered photon in $K$, and $\mathbf{p}^{\prime}$ and $\mathbf{p}_{s}^{\prime}$ to be the corresponding momenta in $K^{\prime}$. We then denote $\Psi^{\prime}$ to be the angle between $\mathbf{p}^{\prime}$ and $\mathbf{p}_{s}^{\prime}, \theta$ to be the angle between $\mathbf{p}$ and $O z, \theta_{s}$ to be the angle between $\mathbf{p}_{s}$ and $O z$, and $\theta^{\prime}$ and $\theta_{s}^{\prime}$ to be the angles between $\mathbf{p}^{\prime}$ and $O z^{\prime}$ and between $\mathbf{p}_{s}^{\prime}$ and $O z^{\prime}$, respectively; $\mu=\cos \theta$ and $\mu^{\prime}=\cos \theta^{\prime}$.

The intensity of gamma-ray emission associated with inverse Compton scattering of photons from the hot spot on primary electrons is equal to

$$
\begin{gathered}
\frac{d N_{s}}{d V d t d \gamma_{s}}=\frac{\pi}{2} r_{e}^{2} c \cdot \frac{n_{e}}{\Gamma^{2}} \\
\times \frac{n_{\gamma}}{\gamma_{c}}\left(2 I_{0}+I_{2}-2 I_{1}+\frac{q^{2}}{q_{c}\left(q+q_{c}\right)} \cdot I_{0}\right),
\end{gathered}
$$

where $\frac{d N_{s}}{d V d t d \gamma_{s}}$ is the number of photons scattered in volume $d V$ in a time $d t$ and possessing after scattering energy $\gamma_{s}$ in the interval $d \gamma_{s}, r_{e}=\frac{e^{2}}{m c^{2}}$ is the classical radius of the electron,

$$
\begin{gathered}
I_{n}=\int_{-1}^{1} \frac{1}{g(\mu)^{n}} f(\mu) \theta\left(g(\mu)-\frac{1}{2}\right) d \mu, \\
g(\mu)=\frac{q_{c}}{q} \Gamma \gamma_{c}(1-\mu), \\
q=\frac{\gamma_{s}}{\Gamma-\gamma_{s}} q_{c}, \quad q_{c}=\frac{1}{2 \Gamma \gamma_{c}\left(1-\mu_{r}\right)} .
\end{gathered}
$$

The function $f(\mu)$ is determined as follows:

$$
\begin{gathered}
f(\mu)=1 \quad \text { for } 0 \leq \theta \leq \theta_{c}-\chi_{d} \text { or } \\
2 \pi-\left(\chi_{d}+\theta_{c}\right) \leq \theta \leq \pi, \\
f(\mu)=0 \quad \text { for } 0 \leq \theta \leq \chi_{d}-\theta_{c} \quad \text { or } \\
\quad \chi_{d}+\theta_{c} \leq \theta \leq \pi, \\
f(\mu)=\frac{1}{\pi} \arccos \left(\frac{\cos \theta_{c}-\mu \cos \chi_{d}}{\sin \chi_{d} \sqrt{1-\mu^{2}}}\right) \text { with } \\
\cos \left(\theta_{c}+\chi_{d}\right) \leq \mu \leq \cos \left(\theta_{c}-\chi_{d}\right) .
\end{gathered}
$$

Here, $\mu=\cos \theta$ and $\chi_{d}$ is the angle between $\mathbf{d}$ and $\mathbf{B}(x)$. In the calculations, we use the value $\mu_{r}=$ $\cos \left(\chi_{d}-\theta_{c}\right)$.

\section{RESULTS}

Figures 1-3 present time-averaged spectra of the gamma-ray emission from the tube of a radio pulsar with $B=0.02 B_{c r}, P=0.2 \mathrm{~s}, \chi=10^{\circ}, k=$ 0.15 , and $\Delta=0.1$. The left-hand plots correspond to $\alpha=\pi$, and the right-hand plots to $\alpha=\pi / 2$. Shown are curves corresponding to a purely dipolar field $(\nu=0$, solid $), \nu=0.1$ (long-dashed), $\nu=0.2$ ( shortdashed) and $\nu=0.3$ (dotted). Figure 1 presents the gamma-ray spectra for the case when the angle between the line of sight and the rotational axis of the pulsar is $10^{\circ}$ (i.e., at some moment in time, the line of sight will be directed parallel to $\mathbf{m}$ ); Figures 2 and 3 present spectra for the cases when this angle is $5^{\circ}$ and $15^{\circ}$, respectively.

The table presents the height of the upper diode plate $z_{c}$, primary-electron energy $\Gamma$ at $\left(z=z_{c}, \xi=0\right.$, $\phi=0$ ), and hot-spot temperature $T$ used to derive the gamma-ray spectra.

Figures 4-8 present the dependence of the gamma-ray intensity of the pulsar tube on the pulsar phase for these same parameters. The angle between the line of sight and the rotational axis is $15^{\circ}$, and phase 0 corresponds to the minimum angle between the line of sight and $\mathbf{m}$. The parameter $\alpha=\pi$ and $\pi / 2$ in the right and left plots, respectively.

We can see from Figs. 1-3 that, with the parameters considered and photon energies $1 \mathrm{MeV}-$ $1 \mathrm{GeV}$, a sharp drop in the intensity of the gammaray curvature radiation is observed in the presence of even a weakly non-dipolar magnetic field $(\nu=0.1)$. The intensity of the gamma-ray emission associated with non-resonance inverse Compton scattering of thermal photons from the polar hot spot on primary electrons does not change so appreciably. The modest variations in the intensity of Compton radiation with increase in the non-dipolarity of the magnetic field (and with the energy of the primary electrons varying by a factor of 20) is due to the fact that, in the cases 

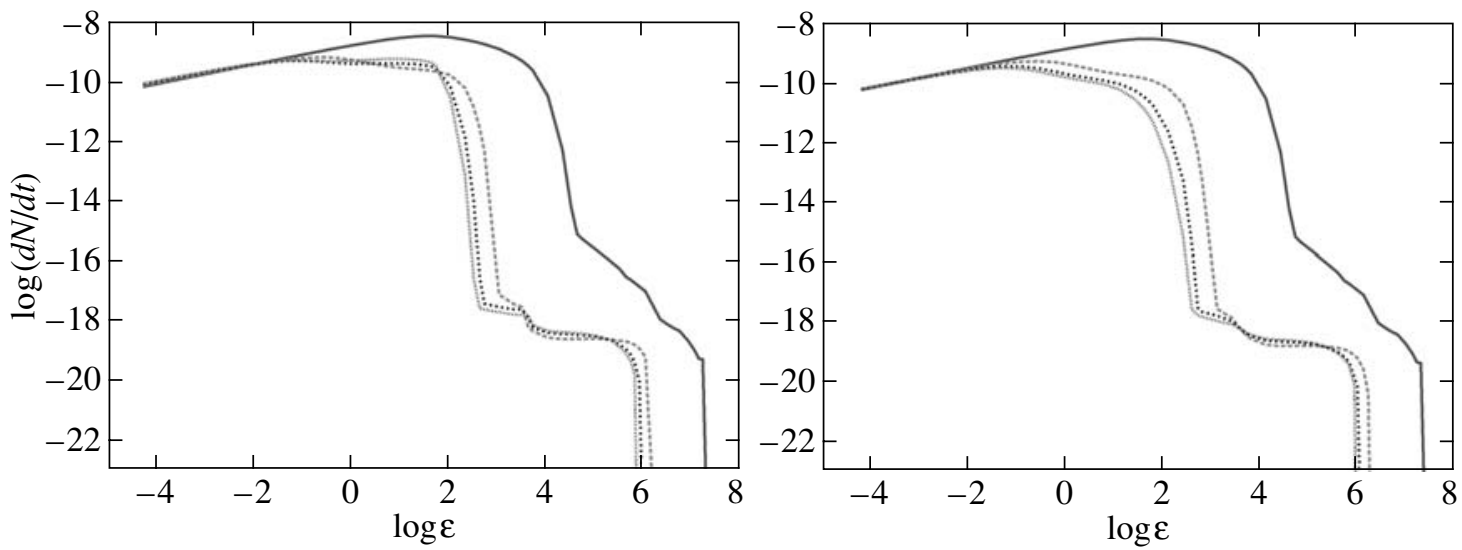

Fig. 1. Time-averaged spectrum of gamma-ray emission from the pulsar tube for $B=0.02 B_{c r}, P=0.2 \mathrm{~s}, \chi=10^{\circ}, k=0.15$, $\Delta=0.1$. Shown are curves corresponding to a purely dipolar field $(\nu=0$, solid), $\nu=0.1$ (long-dashed), $\nu=0.2$ (shortdashed) and $\nu=0.3$ (dotted); the angle between the line of sight and the rotational axis of the pulsar is $10^{\circ}$. The left plot corresponds to $\alpha=\pi$ and the right plot to $\alpha=\pi / 2$. The intensity of gamma-ray emission $\frac{d N}{d t}$ is measured via the flux from the source received at a distance of $1 \mathrm{kpc}$ in photons $\mathrm{cm}^{-2} \mathrm{~s}^{-1} \mathrm{MeV}^{-1}$. The photon energy $\epsilon$ is measured in $\mathrm{MeV}$.
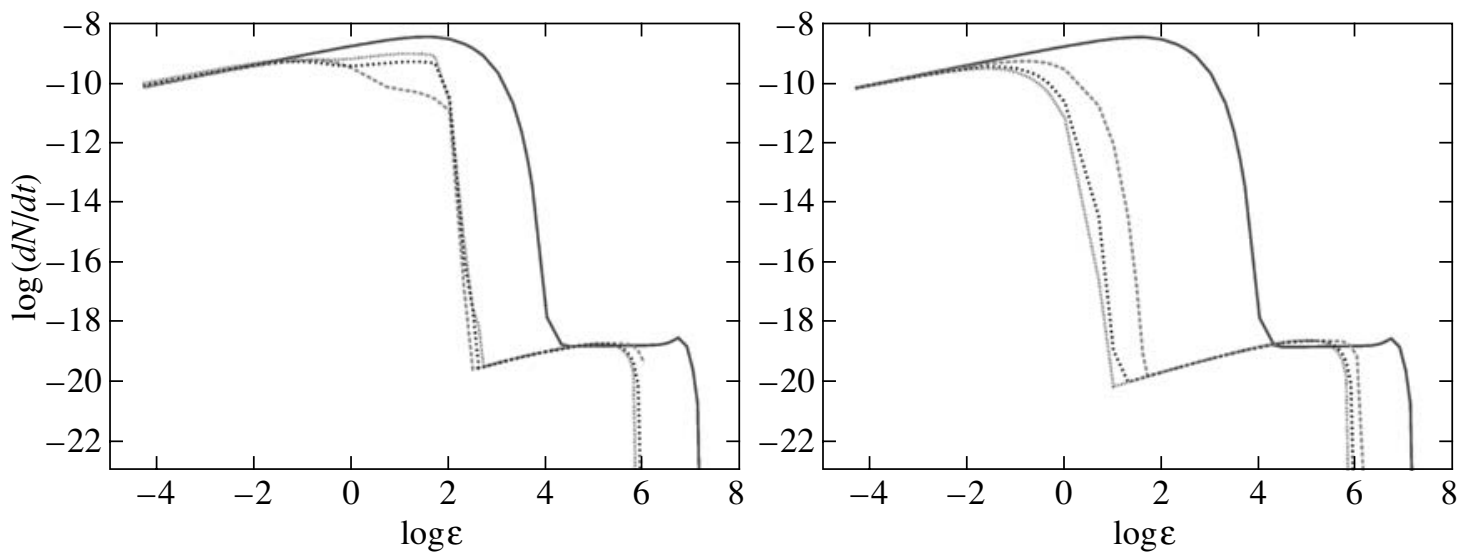

Fig. 2. Same as Fig. 1 for the case when the angle between the line of sight and the rotational axis of the pulsar is $5^{\circ}$.
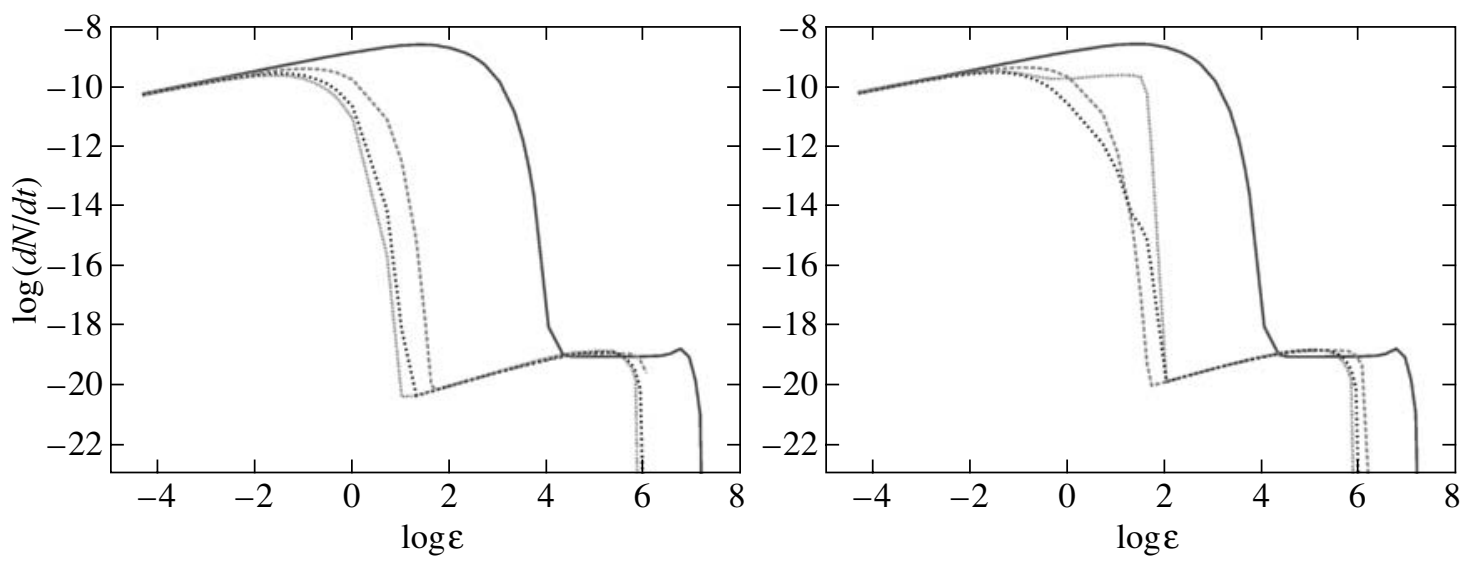

Fig. 3. Same as Fig. 1 for the case when the angle between the line of sight and the rotational axis of the pulsar is $15^{\circ}$. 

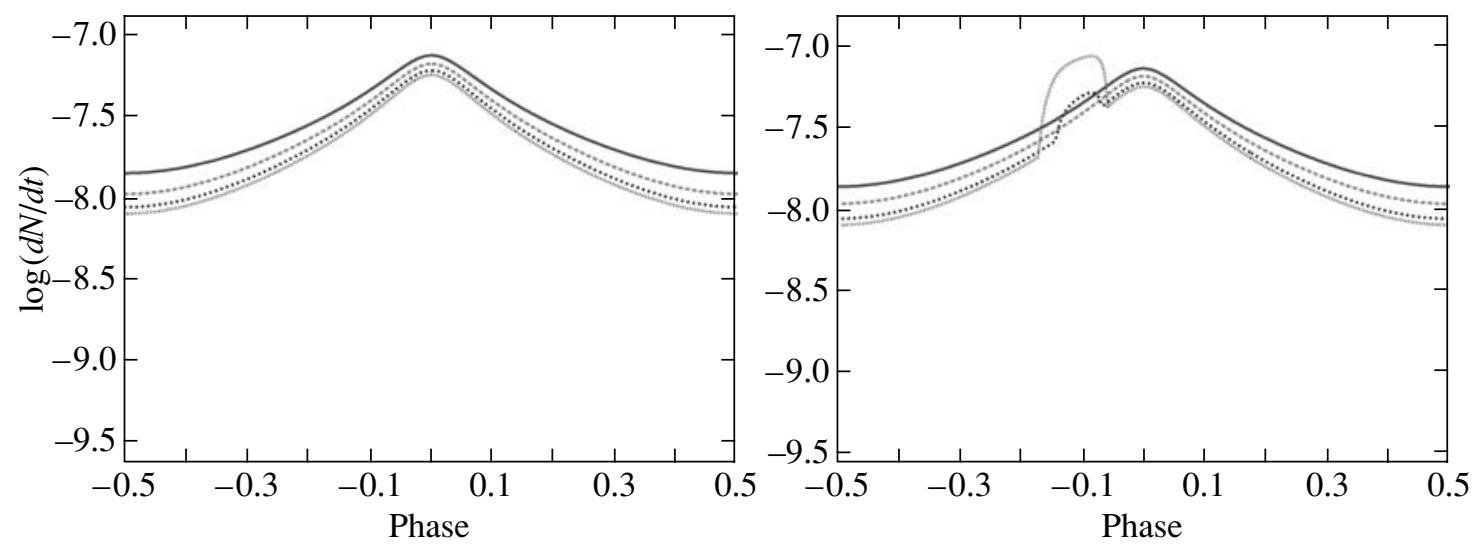

Fig. 4. Shape of a pulse of gamma-ray emission from a pulsar tube with $B=0.02 B_{c r}, P=0.2 \mathrm{~s}, \chi=10^{\circ}, k=0.15$, and $\Delta=0.1$. Shown are curves corresponding to a purely dipolar field $(\nu=0$, solid), $\nu=0.1$ (long-dashed), $\nu=0.2$ (shortdashed) and $\nu=0.3$ (dotted); the angle between the line of sight and the rotational axis of the pulsar is $15^{\circ}$. The left plot corresponds to $\alpha=\pi$ and the right plot to $\alpha=\pi / 2$. The intensity of gamma-ray emission $\frac{d N}{d t}$ is measured via the flux from the source received at a distance of $1 \mathrm{kpc}$ in units of photons $\mathrm{cm}^{-2} \mathrm{~s}^{-1} \mathrm{MeV}^{-1}$. The photon energy is $10 \mathrm{keV}$.
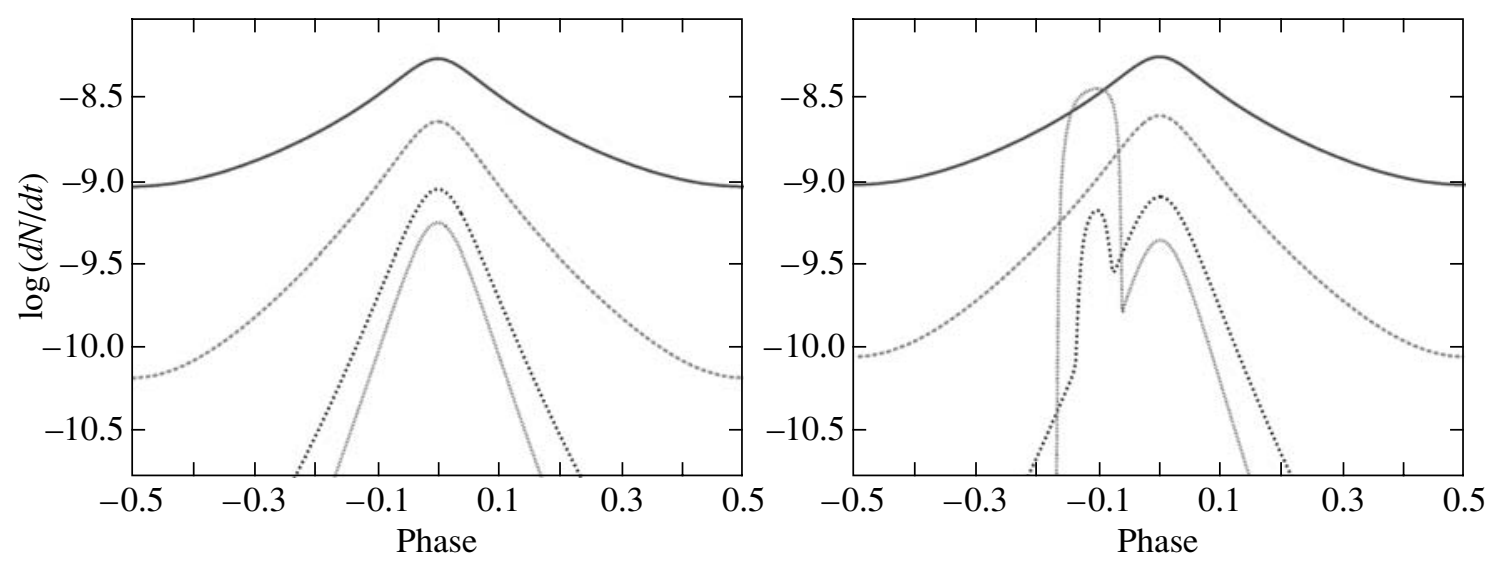

Fig. 5. Same as Fig. 4 for a photon energy of $500 \mathrm{keV}$.
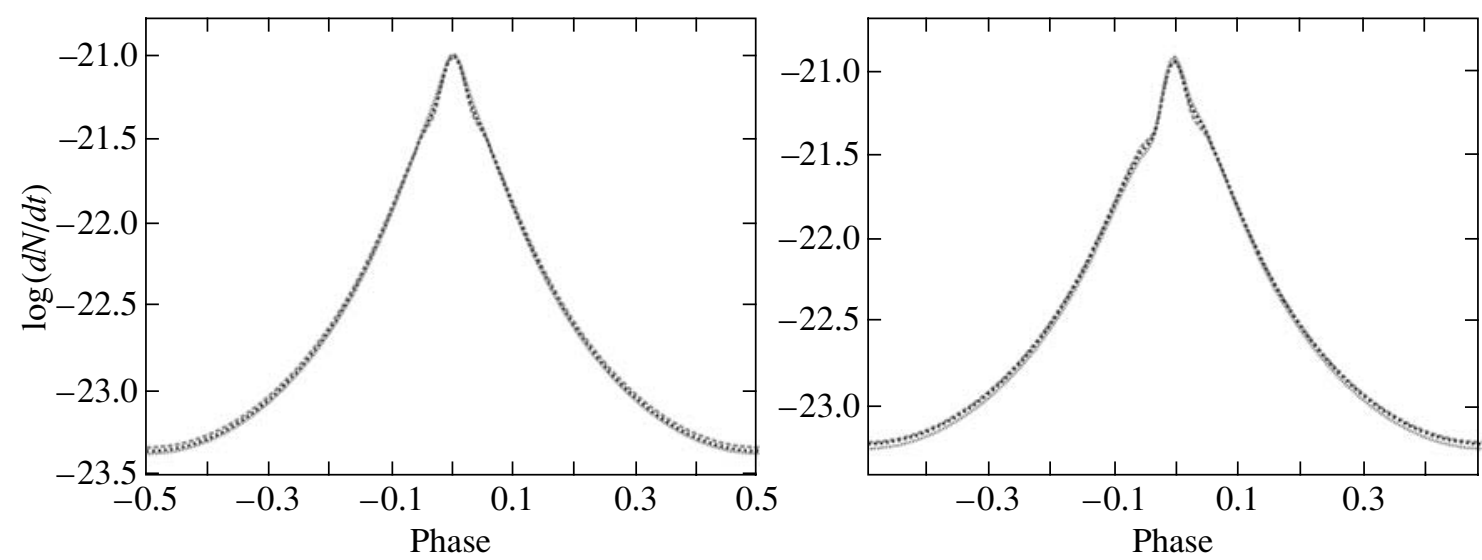

Fig. 6. Same as Fig. 4 for a photon energy of $100 \mathrm{MeV}$. 

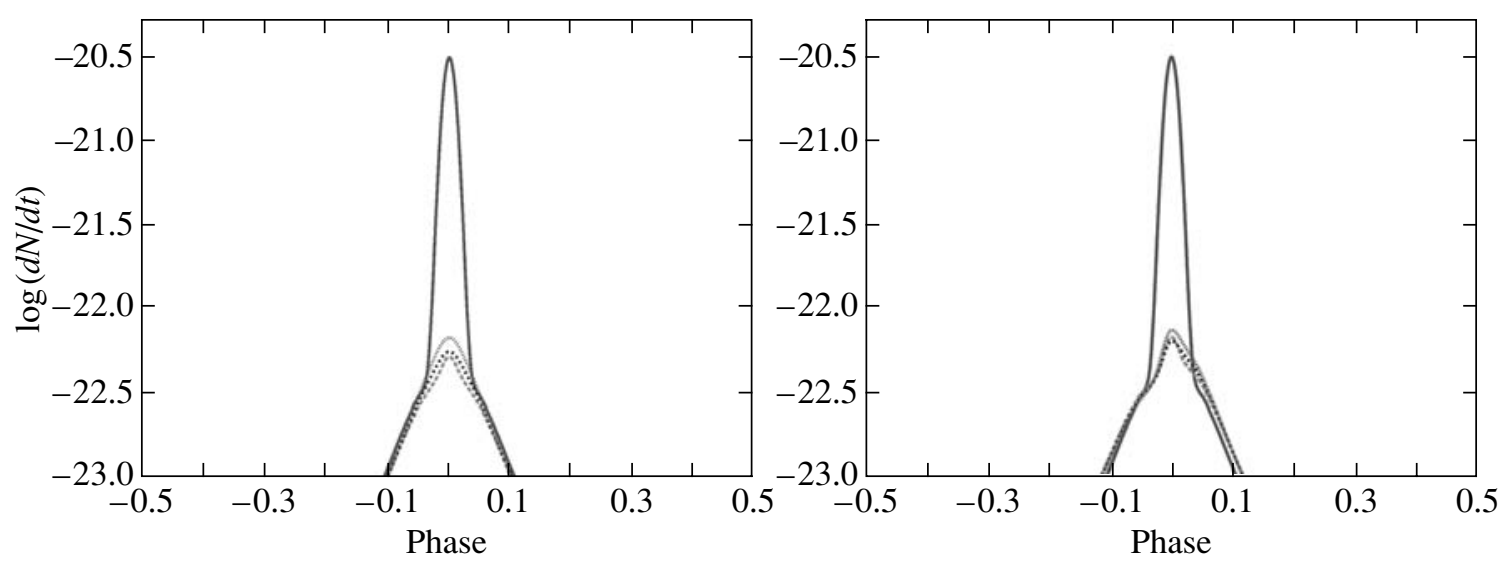

Fig. 7. Same as Fig. 4 for a photon energy of $10 \mathrm{GeV}$.
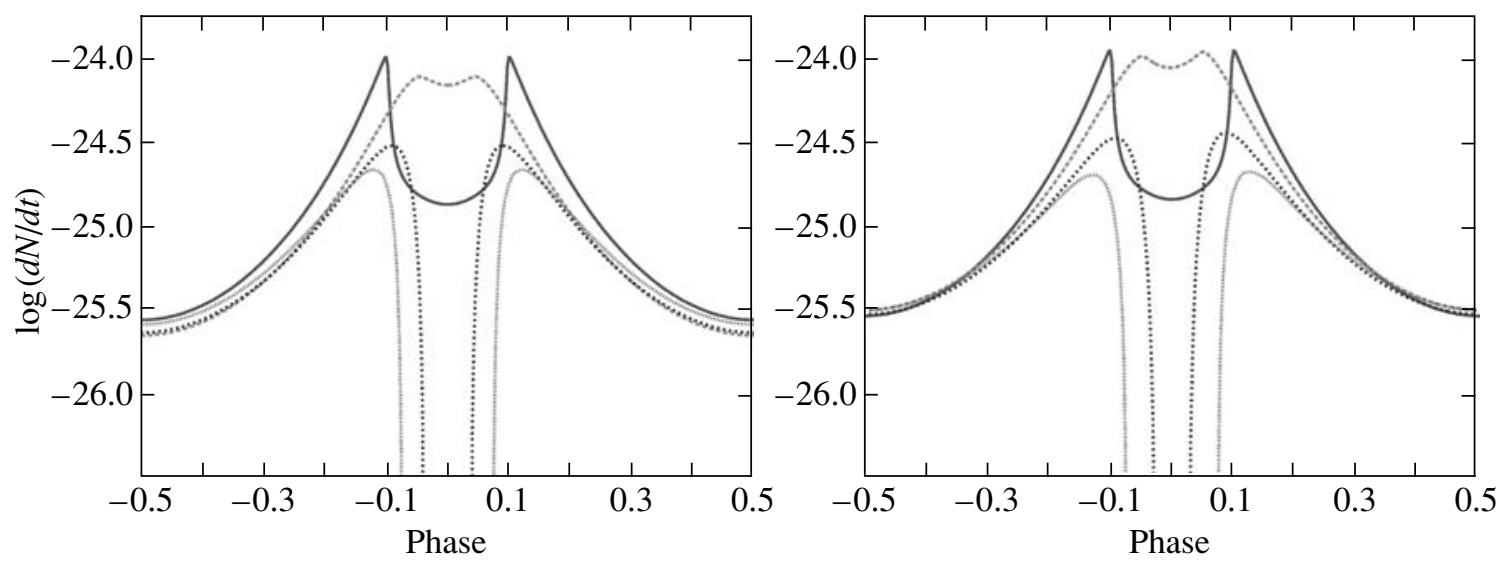

Fig. 8. Same as Fig. 4 for a photon energy of $500 \mathrm{GeV}$.

considered and with a specified photon energy $\gamma_{s}$, the main contribution to non-resonance Compton radiation is made by regions where $q \sim 0.1-1$. Note that, since photons with energies $\gamma_{s} \sim 10^{3}-10^{5}$ begin to freely leave the pulsar magnetosphere only from heights of the order of $\eta \sim 20-100$, the structure of the magnetic field near the surface of the neutron star influences them only weakly.

We especially wish to make note of the case presented in Fig. 1, when we can observe photons traveling along the axis of the main dipole $\mathbf{m}$. In the case $\nu=0$, the main contribution to non-resonance Compton radiation is made by regions with $\eta \sim 1-2$, and a sharp peak with a width of less than $10^{-3}$ in phase is formed near phase 0 . It is precisely this peak that makes the main contribution to the average intensity of the Compton radiation. Unfortunately, the calculated intensity of the radiation at the peak has large uncertainty, but this peak provides an increase in the intensity of the Compton radiation by at least an order of magnitude (compared with the non-dipolar case). In the case of a non-dipolar magnetic field $(\nu=$
$0.1,0.2,0.3)$ and at energies $\gamma_{s} \sim 10^{2}-10^{3}$, the main contribution to the radiation is made by regions at the edges of the pulsar tube, where the electron energy $\Gamma$ is comparable to the photon energy $\gamma_{s}$, and $q \sim$ $0.1-1$, forming two narrow peaks in the phase profile. At photon energies $\gamma_{s} \sim 10^{3}-10^{5}$, the contributions of these peaks to the average intensity of the Compton radiation decrease, while the contribution of photons emerging along the main dipole axis increases. At photon energies $\gamma_{s}$ comparable to the maximum energies of primary electrons in the tube, it becomes difficult to produce a similar photon during scattering on an electron of a thermal photon traveling at a small angle to the electron momentum. Because of this, the contribution to the mean radiation intensity made by regions lying closer to the edges of the pulsar tube begins to grow.

The slight asymmetry of the spectra about phase 0 for $\alpha=\pi / 2$ in Figs. 7 and 8 is due to the fact that, as the phase varies, the angle at which the polar hot spot is viewed (in regions making the largest contribution at a given phase) also varies slightly. 
We note that including scattering of hard Compton photons on thermal photons from the hot spot could lead to a decrease in the intensity of the central peak, which could, in principle, strongly decrease the intensity of Compton radiation for a purely dipolar field $(\nu=0)$ for the case shown in Fig. 1.

\section{ACKNOWLEDGMENTS}

The authors thank V.D. Pal'shin and M.E. Gusakov for help with the numerical calculations; M.E. Gusakov, M.V. Ulanov, and A.I. Chugunov for their support and valuable comments; and A.N. Timokhin for his support of this work. This work was financially supported by the Russian Foundation for Basic Research (project no. 04-02-17590) and the Program of Support for Leading Scientific Schools of the Russian Federation (grant no. NSh1115.2003.2).

\section{REFERENCES}

1. V. Urpin and J. Gil, Astron. Astrophys. 415, 305 (2004).

2. U. Geppert, M. Rheinhardt, and J. Gil, Astron. Astrophys. 412, L33 (2003).

3. E. Asseo and D. Khechinashvili, Mon. Not. R. Astron. Soc. 334, 743 (2002).

4. A. N. Timokhin, G. S. Bisnovatyi-Kogan, and H. C. Spruit, Mon. Not. R. Astron. Soc. 316, 734 (2000).

5. J. Gil, G. Melikidze, and B. Zhang, astro-ph/0601613 (2006).
6. J. A. Hibschman and J. Arons, Astrophys. J. 554, 624 (2001).

7. A. K. Harding and A. G. Muslimov, Astrophys. J. 556, $987(2001)$.

8. A. K. Harding and A. G. Muslimov, Astrophys. J. 568, $862(2002)$.

9. K. Hirotani, A. K. Harding, and S. Shibata, Astrophys. J. 591, 334 (2003).

10. A. K. Harding, V. V. Usov, and A. Muslimov, astro-ph 0510135v1 (2005).

11. E. T. Scharlemann, J. Arons, and W. M. Fawley, Astrophys. J. 222, 297 (1978).

12. J. Gil and D. Mitra, Astrophys. J. 550, 383 (2001).

13. D. P. Barsukov, E. M. Kantor, and A. I. Tsygan, Astron. Zh. 83, 184 (2006) [Astron. Rep. 50, 159 (2006)].

14. V. D. Pal'shin and A. I. Tsygan, Astron. Zh. 73, 426 (1996) [Astron. Rep. 40, 385 (1996)].

15. V. D. Pal'shin and A. I. Tsygan, Preprint No. 1718, FTI (Ioffe Physicotechnical Institute, St. Petersburg, 1998).

16. A. I. Tsygan, in Pulsar Astronomy-2000 and Beyond, Ed. by M. Kramer, N. Wex, and R. Wielebinski, Astron. Soc. Pac. Conf. Ser. 202, 473 (2000).

17. E. M. Kantor and A. I. Tsygan, Astron. Zh. 80, 665 (2003) [Astron. Rep. 47, 613 (2003)].

18. A. G. Muslimov and A. I. Tsygan, Mon. Not. R. Astron. Soc. 255, 61 (1992).

19. F. C. Jones, Phys. Rev. 167, 1159 (1968).

20. J. Gil and G. I. Melikidze, Astrophys. J. 577, 909 (2002).

Translated by D. Gabuzda 